\title{
CONDIÇÕES LMI DO TEOREMA DO GANHO PEQUENO ESCALONADO PARA ANÁLISE DE ESTABILIDADE DE SISTEMAS INCERTOS COM ATRASO
}

\author{
Giórgio Valmórbida* \\ giorgio@dt.fee.unicamp.br
}

\author{
Valter Júnior de Souza Leite ${ }^{\dagger}$ \\ valterdieee.org
}

\author{
Pedro Luis Dias Peres* \\ peresddt.fee.unicamp.br
}

${ }^{*}$ Faculdade de Engenharia Elétrica e de Computação, Universidade Estadual de Campinas, CP 6101, 13083-970, Campinas SP - Brasil.

${ }^{\dagger}$ UnED Divinópolis - CEFET-MG, R. Monte Santo, 319 - 35502-036, Divinópolis - MG - Brasil.

\section{RESUMO}

A equivalência entre resultados para análise de estabilidade baseados no método de Lyapunov de sistemas com atraso precisamente conhecidos e resultados de estabilidade robusta de sistemas de comparação sem atraso, empregando condições do teorema do ganho pequeno escalonado, foi apresentada recentemente na literatura. A extensão desses resultados para tratar sistemas com atraso e incerteza em domínios politópicos é a principal contribuição deste artigo. A partir da definição de uma realização genérica para um sistema, são apresentadas desigualdades matriciais lineares com variáveis extras, equivalentes a condições de ganho pequeno escalonadas. Devido à inserção dessas variáveis extras, condições independentes e dependentes do atraso para análise de estabilidade de sistemas com atraso em domínios politópicos podem ser elaboradas empregando matrizes de Lyapunov dependentes de parâmetro, resultando em condições menos conservadoras que outras já apresentadas na literatura, como ilustrado por exemplos numéricos.

PALAVRAS-CHAVE: Estabilidade robusta, teorema do ganho pequeno, sistemas com atraso, desigualdades matriciais

Artigo submetido em 02/06/2005

1a. Revisão em 07/08/2006

2a. Revisão em 04/07/2007

Aceito sob recomendação do Editor Associado

Prof. Liu Hsu lineares.

\section{ABSTRACT}

Recently, it has been shown that some Lyapunov-based stability conditions for precisely known time-delay systems are equivalent to the robust stability of a delay-free comparison system through the small gain theorem with constant scales. The extension of those previous results to cope with uncertain time-delay systems in polytopic domains is the main contribution of this paper. From the definition of a generic system realization, linear matrix inequalities that are equivalent to the scaled small gain conditions but have extra matrix variables are given. Thanks to these extra matrices, delay-independent and delay-dependent stability conditions can be obtained for the analysis of time-delay systems in polytopic domains through parameter-dependent Lyapunov matrices, yielding conditions that are less conservative than others in the literature, as illustrated by means of numerical examples.

KEYWORDS: Robust stability, small gain theorem, timedelay systems, linear matrix inequalities. 


\section{INTRODUÇÃO}

A análise de estabilidade de sistemas com atraso de transporte é um tema que vem sendo bastante explorado, o que pode ser visto a partir do grande número de trabalhos publicados recentemente (ver Gu et al. (2003), Niculescu (2001), Mahmoud (2000) e suas referências). Duas abordagens possíveis na análise de estabilidade de sistemas com atraso são: estabilidade independente do atraso e estabilidade dependente do atraso (Richard, 2003). Na primeira, o interesse consiste em determinar se o sistema é estável independentemente da magnitude do atraso. A segunda abordagem se preocupa em definir a máxima magnitude do atraso tal que o sistema permaneça estável. As condições para análise de estabilidade podem ser formuladas no domínio do tempo ou no domínio da frequiência, mas sobretudo devem ser eficientes e numericamente tratáveis. Para atender a esses requisitos, técnicas no domínio do tempo utilizando funcionais de Lyapunov-Krasovskii são amplamente empregadas na análise de estabilidade de sistemas com atraso, gerando condições suficientes para a estabilidade do sistema formuladas como desigualdades matriciais lineares (linear matrix inequalities em inglês LMIs) que podem ser resolvidas com algoritmos de tempo polinomial (Gahinet et al., 1995).

Uma conexão entre condições baseadas em funções de Lyapunov para análise de estabilidade de sistemas contínuos com atraso precisamente conhecidos e condições no domínio da frequiência do teorema do ganho pequeno escalonado aplicadas a um sistema de comparação sem atrasos foi apresentada em Zhang et al. (2001) (ver também Huang e Zhou (1999) e Niculescu e Chen (1999)). Partindo de uma representação genérica para um sistema, essas condições reproduzem alguns resultados já conhecidos para análise de sistemas contínuos com atraso (Li e de Souza, 1995), (Niculescu et al., 1995) e (Verriest et al., 1993), obtidos a partir de funcionais de Lyapunov-Krasovskii e formulados como LMIs por meio de uma escolha adequada das matrizes do sistema.

A presença de incerteza nos parâmetros do sistema torna a análise de estabilidade uma tarefa mais complexa. Uma maneira de tratar as incertezas paramétricas na análise de sistemas com atraso é utilizar matrizes constantes nos funcionais de Lyapunov-Krasovskii. Entretanto, essa técnica, conhecida como estabilidade quadrática, pode levar a resultados muito conservadores.

Resultados menos conservadores para estabilidade robusta foram obtidos recentemente por meio do uso de funções de Lyapunov dependentes de parâmetro e alguns desses resultados foram estendidos para tratar sistemas com atraso. É importante destacar que as condições LMI obtidas em de Oliveira et al. (1999), Leite e Peres (2003), Peaucelle et al. (2000) para estabilidade robusta reduzem o conservadorismo na análise de estabilidade graças à presença de variáveis extras e funcionais de Lyapunov dependentes de parâmetro.

Este artigo trata da análise de estabilidade independente e dependente do atraso de sistemas contínuos com atraso e parâmetros incertos representados em politopos utilizando as condições LMI de ganho pequeno escalonado. O atraso e os parâmetros do sistema são considerados invariantes no tempo. Condições suficientes na forma de LMIs, independentes e dependentes do atraso, são formuladas em termos dos vértices do politopo, estendendo assim os resultados da literatura para o caso incerto, introduzindo também novas condições LMI com variáveis extras.

Uma solução factível para as LMIs garante a existência de matrizes dependentes de parâmetro usadas no funcional de Lyapunov-Krasovskii que assegura a estabilidade robusta. Mostra-se, por meio de exemplos numéricos, que as novas condições com matrizes extras reduzem o conservadorismo na análise de estabilidade robusta independente e dependente do atraso de sistemas incertos contínuos com atraso.

\section{Notação}

O símbolo $\left({ }^{\prime}\right)$ indica a transposta da matriz; $P>0$ significa que $P$ é simétrica definida positiva. $N$ representa o número de vértices de um politopo. $\mathbb{R}_{+}$é o conjunto dos números reais não negativos e $\mathbb{C}$ é o conjunto dos números complexos. I e 0 representam, respectivamente, as matrizes identidade e nula de dimensões adequadas. $\mathscr{C}_{\tau}=\mathscr{C}\left([-\tau, 0], \mathbb{R}^{n}\right)$ denota o espaço de Banach de funções vetoriais contínuas mapeando o intervalo $[-\tau, 0]$ em $\mathbb{R}^{n}$ com a topologia de convergência uniforme. $\|\cdot\|$ se refere à norma vetorial euclidiana. $\|\phi\|_{c}=$ $\sup _{-\tau \leq t \leq 0}\|\phi(t)\|$ representa a norma de uma função $\phi \in \mathscr{C}_{\tau}$. Quando o atraso for finito "sup" pode ser substituído por "max". $\mathscr{C}_{\tau}^{v}$ é o conjunto definido por $\mathscr{C}_{\tau}^{v}=\left\{\phi \in \mathscr{C}_{\tau} ; \|\right.$ $\left.\phi \|_{c}<v, v>0\right\}$. O símbolo $\star$ representa blocos simétricos nas LMIs.

\section{PRELIMINARES}

Considere o sistema contínuo dado por:

$$
\dot{x}(t)=A x(t)+A_{\tau} x(t-\tau)
$$

cujas condições iniciais são

$$
x\left(t_{0}+\theta\right)=\phi(\theta), \forall \theta \in[-\tau, 0], t_{0}, \phi \in \mathbb{R}_{+} \times \mathscr{C}_{\tau}^{\nu}
$$

sendo que $x \in \mathbb{R}^{n}$ é o estado e $\tau>0$ é um atraso de transporte constante.

O sistema com atraso (1)-(2) foi analisado em (Zhang et al., 2001) pela definição de um sistema de comparação com uma 


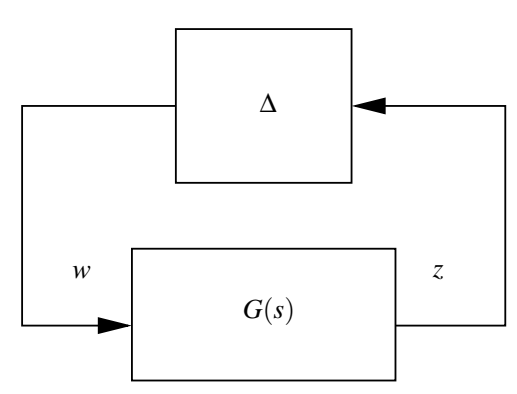

Figura 1: Sistema $G(s)$ afetado pela incerteza $\Delta$.

variável matricial livre, reescrito como a interconexão de um sistema linear invariante no tempo $G(s)$ com um bloco $\Delta$ representando a incerteza estruturada, como mostrado na Figura 1. Desse modo, a estabilidade robusta do sistema afetado pela incerteza estruturada $\Delta,\|\Delta\|_{\infty} \leq 1$, garante a estabilidade do sistema (1)-(2), como descrito no lema a seguir. Maiores detalhes sobre essa equivalência podem também ser obtidos em Valmórbida (2006).

Lema 1 Considere o sistema interconectado mostrado na Figura 1 com $\Delta=\operatorname{diag}\left\{\Delta_{1}, \ldots, \Delta_{r}\right\}, \Delta_{i}=\lambda_{i} \mathbf{I}_{n_{i}}, \lambda_{i} \in \mathbb{C}, i=$ $1, \ldots, r,\|\Delta\|_{\infty} \leq 1$ e $G(s)$ com realização mínima dada por

$$
\left\{\begin{array}{l}
\dot{x}=\mathscr{A} x+\mathscr{B} w \\
z=\mathscr{C} x+\mathscr{D} w \\
w=\Delta z
\end{array}\right.
$$

O sistema em malha fechada

$$
\dot{x}=\left(\mathscr{A}+\mathscr{B} \Delta(\mathbf{I}-\Delta \mathscr{D})^{-1} \mathscr{C}\right) x
$$

é estável se existirem matrizes $P=P^{\prime}>0, P \in \mathbb{R}^{n \times n}$, e $Q=$ $\operatorname{diag}\left\{Q_{1}, \ldots, Q_{r}\right\}>0, Q_{i} \in \mathbb{R}^{n_{i} \times n_{i}}, i=1, \ldots, r$ de dimensões apropriadas tais que

$$
\left[\begin{array}{ccc}
\mathscr{A}^{\prime} P+P \mathscr{A} & P \mathscr{B} & \mathscr{C}^{\prime} Q \\
\star & -Q & \mathscr{D}^{\prime} Q \\
\star & \star & -Q
\end{array}\right]<0
$$

No lema que segue são propostas condições LMI equivalentes às obtidas no Lema 1 , porém com variáveis matriciais extras.

Lema 2 Existem matrizes $P=P^{\prime}>0, P \in \mathbb{R}^{n \times n}$, e $Q=$ $\operatorname{diag}\left\{Q_{1}, \ldots, Q_{r}\right\}>0, Q_{i} \in \mathbb{R}^{n_{i} \times n_{i}}, i=1, \ldots, r$ tais que (4) no Lema 1 é satisfeita se e somente se existirem $P=P^{\prime}>0$, $Q=\operatorname{diag}\left\{Q_{1}, \ldots, Q_{r}\right\}>0, Q_{i} \in \mathbb{R}^{n_{i} \times n_{i}}, i=1, \ldots, r$ e $\mathscr{X}$ de dimensões apropriadas tais que

$$
\tilde{Q}+\mathscr{X} \tilde{B}+\tilde{B}^{\prime} \mathscr{X}^{\prime}<0
$$

com

$$
\tilde{Q}=\left[\begin{array}{cccc}
\mathbf{0} & P & \mathbf{0} & \mathbf{0} \\
\star & \mathbf{0} & \mathbf{0} & \mathbf{0} \\
\star & \star & Q & \mathbf{0} \\
\star & \star & \star & -Q
\end{array}\right]
$$

$$
\tilde{B}=\left[\begin{array}{llll}
\mathbf{I} & -\mathscr{A} & \mathbf{0} & -\mathscr{B} \\
\mathbf{0} & -\mathscr{C} & \mathbf{I} & -\mathscr{D}
\end{array}\right]
$$

Prova: Usando o complemento de Schur (Boyd et al., 1994), pode ser mostrado que (4) é equivalente a

$$
\Omega=\left[\begin{array}{cc}
\mathscr{A}^{\prime} P+P \mathscr{A}+\mathscr{C}^{\prime} Q \mathscr{C} & P \mathscr{B}+\mathscr{C}^{\prime} Q \mathscr{D} \\
\star & -Q+\mathscr{D}^{\prime} Q \mathscr{D}
\end{array}\right]<0
$$

e, portanto,

$$
\begin{aligned}
{\left[\begin{array}{ll}
x^{\prime} & w^{\prime}
\end{array}\right] \Omega\left[\begin{array}{ll}
x^{\prime} & w^{\prime}
\end{array}\right]^{\prime}=} \\
=\left(x^{\prime} \mathscr{A}^{\prime}+w^{\prime} \mathscr{B}^{\prime}\right) P x+x^{\prime} P(\mathscr{A} x+\mathscr{B} w) \\
\quad+\left(x^{\prime} \mathscr{C}^{\prime}+w^{\prime} \mathscr{D}^{\prime}\right) Q(\mathscr{C} x+\mathscr{D} w)-w^{\prime} Q w<0
\end{aligned}
$$

Agora, usando (3) e definindo $\zeta=\left[\begin{array}{cccc}\dot{x}^{\prime} & x^{\prime} & z^{\prime} & w^{\prime}\end{array}\right]^{\prime}$, obtém-se $\tilde{B} \zeta=0$ com $\tilde{B}$ dado por (7) e $\dot{x}^{\prime} P x+x^{\prime} P \dot{x}+z^{\prime} Q z-$ $w^{\prime} Q w=\zeta^{\prime} \tilde{Q} \zeta$ com $\tilde{Q}$ dada por (6). Finalmente, usando resultados do Lema de Finsler de Oliveira e Skelton (2001), existe $\tilde{Q}$ tal que $\zeta^{\prime} \tilde{Q} \zeta<0, \forall \zeta: \tilde{B} \zeta=0$ se e somente se existirem $\tilde{Q}$ e $\mathscr{X}$ tais que a equação (5) é satisfeita.

As condições apresentadas no Lema 2 são equivalentes às condições dadas no Lema 1 quando $\mathscr{A}, \mathscr{B}, \mathscr{C}$, and $\mathscr{D}$ são matrizes precisamente conhecidas. Entretanto, as variáveis matriciais introduzidas proporcionam graus de liberdade úteis que podem ser usados na análise de sistemas incertos em domínios politópicos, como apresentado em Ramos e Peres (2001), Ramos e Peres (2002), Leite e Peres (2003).

Nas próximas seções será mostrado como as escolhas particulares para $(\mathscr{A}, \mathscr{B}, \mathscr{C}, \mathscr{D})$ usadas em Zhang et al. (2001) também podem ser aplicadas às condições do Lema 2. Além disso, são apresentadas condições LMI dependentes de parâmetro assegurando a estabilidade robusta do sistema (1)(2) com matrizes incertas $\left(A, A_{\tau}\right)$ pertencendo a um domínio politópico. Como em Zhang et al. (2001), as condições são apresentadas para sistemas com apenas um atraso, mas são facilmente extensíveis para analisar estabilidade de sistemas com múltiplos atrasos. Embora todas as escolhas para $(\mathscr{A}, \mathscr{B}, \mathscr{C}, \mathscr{D})$ apresentadas Zhang et al. (2001) pudessem ser aplicadas equivalentemente às condições do Lema 2 , apenas a condição independente do atraso e duas condições de estabilidade dependentes do atraso são consideradas neste artigo.

\section{RESULTADOS PRINCIPAIS}

Em (Zhang et al., 2001), escolhas particulares de $(\mathscr{A}, \mathscr{B}, \mathscr{C}, \mathscr{D})$ reproduzem algumas condições da literatura para estabilidade do sistema (1)-(2). Três dessas escolhas (levando às condições apresentadas em Verriest et al. (1993), Li e de Souza (1995) e Park (1999)) são exploradas neste artigo empregando o Lema 2, levando a condições 
equivalentes com variáveis extras. De posse dessas condições, o objetivo é estendê-las para o caso de matrizes incertas $\left(A, A_{\tau}\right)$ pertencentes a um domínio politópico.

\subsection{Sistemas Precisamente Conhecidos}

Três condições suficientes para análise de estabilidade do sistema (1)-(2) são obtidas com escolhas especiais de $(\mathscr{A}, \mathscr{B}, \mathscr{C}, \mathscr{D})$ aplicadas ao Lema 2 , apresentadas nos lemas a seguir.

Lema 3 O sistema (1)-(2) é assintóticamente estável independentemente do valor do atraso se existirem $P=P^{\prime}>$ $0, Q=Q^{\prime}>0, F_{i}, G_{i}, i=1, \ldots, 4$, matrizes do $\mathbb{R}^{n \times n}$, tais que (8) é satisfeita.

Prova: Em Zhang et al. (2001), mostra-se que a escolha

$$
\mathscr{A}=A, \mathscr{B}=A_{\tau}, \mathscr{C}=\mathbf{I}, \mathscr{D}=\mathbf{0}
$$

substituída no Lema 1 resulta em condições suficientes para a estabilidade do sistema (1)-(2) independentemente do valor do atraso. A mesma escolha aplicada ao Lema 2 leva às LMIs do Lema 3, com $\tilde{Q}$ descrito por (6) e

$$
\mathscr{X}=\left[\begin{array}{llll}
F_{1}^{\prime} & F_{2}^{\prime} & F_{3}^{\prime} & F_{4}^{\prime} \\
G_{1}^{\prime} & G_{2}^{\prime} & G_{3}^{\prime} & G_{4}^{\prime}
\end{array}\right]^{\prime}
$$

Lema 4 O sistema (1)-(2) é assintóticamente estável para $0 \leq \tau \leq \bar{\tau}$ se existirem $P=P^{\prime}>0, V=V^{\prime}>0, U=U^{\prime}>$ $0, F_{i}, G_{i}, H_{i}, i=1, \ldots, 6$, matrizes do $\mathbb{R}^{n \times n}$, tais que (11) é satisfeita, com

$$
\begin{gathered}
\Psi_{1}=-F_{1}\left(A+A_{\tau}\right)-G_{1} A-H_{1} A_{\tau}+F_{2}^{\prime}+P \\
\Psi_{2}=-F_{2}\left(A+A_{\tau}\right)-G_{2} A-H_{3} A_{\tau}-\left(A^{\prime}+A_{\tau}^{\prime}\right) F_{2}^{\prime} \\
-A^{\prime} G_{2}^{\prime}-A_{\tau}^{\prime} H_{3}^{\prime} \\
\Psi_{3}=G_{2}-\left(A^{\prime}+A_{\tau}^{\prime}\right) F_{3}^{\prime}-A^{\prime} G_{3}^{\prime}-A_{\tau}^{\prime} H_{3}^{\prime} \\
\Psi_{4}=H_{2}-\left(A^{\prime}+A_{\tau}^{\prime}\right) F_{4}^{\prime}-A^{\prime} G_{4}^{\prime}-A_{\tau}^{\prime} H_{4}^{\prime} \\
\Psi_{5}=-\bar{\tau} F_{2} A_{\tau}-\left(A^{\prime}+A_{\tau}^{\prime}\right) F_{5}^{\prime}-A^{\prime} G_{5}^{\prime}-A_{\tau}^{\prime} H_{5}^{\prime} \\
\Psi_{6}=-\bar{\tau} F_{2} A_{\tau}-\left(A^{\prime}+A_{\tau}^{\prime}\right) F_{6}^{\prime}-A^{\prime} G_{6}^{\prime}-A_{\tau}^{\prime} H_{6}^{\prime}
\end{gathered}
$$

Prova: Em Zhang et al. (2001), mostra-se que a escolha

$$
\mathscr{A}=A+A_{\tau}, \mathscr{B}=\left[\begin{array}{ll}
\bar{\tau} A_{\tau} & \bar{\tau} A_{\tau}
\end{array}\right], \mathscr{C}=\left[\begin{array}{c}
A \\
A_{\tau}
\end{array}\right], \mathscr{D}=\mathbf{0}
$$

substituída no Lema 1 resulta em condições suficientes para a estabilidade do sistema (1)-(2) para $0 \leq \tau \leq \bar{\tau}$. A mesma escolha aplicada ao Lema 2 leva às LMIs do Lema $4, \operatorname{com} \tilde{Q}$ descrito por (6) e

$$
Q=\operatorname{diag}\{V, U\}
$$

$$
\mathscr{X}=\left[\begin{array}{llllll}
F_{1}^{\prime} & F_{2}^{\prime} & F_{3}^{\prime} & F_{4}^{\prime} & F_{5}^{\prime} & F_{6}^{\prime} \\
G_{1}^{\prime} & G_{2}^{\prime} & G_{3}^{\prime} & G_{4}^{\prime} & G_{5}^{\prime} & G_{6}^{\prime} \\
H_{1}^{\prime} & H_{2}^{\prime} & H_{3}^{\prime} & H_{4}^{\prime} & H_{5}^{\prime} & H_{6}^{\prime}
\end{array}\right]^{\prime}
$$

Lema 5 O sistema (1)-(2) é assintóticamente estável para $0 \leq \tau \leq \bar{\tau}$ se existirem $P=P^{\prime}>0, V=V^{\prime}>0, U=U^{\prime}>$ $0, N_{i}, F_{i}, G_{i}, H_{i}, i=1, \ldots, 6$, matrizes do $\mathbb{R}^{n \times n}$, tais que a equação (14) é satisfeita, com

$$
\begin{gathered}
\Phi_{1}=-F_{1} A-\left(F_{1}+N_{1}\right) A_{\tau}-G_{1} A_{\tau} A-H_{1}+F_{2}^{\prime}+P \\
\Phi_{2}=-F_{2} A-A^{\prime} F_{2}^{\prime}-\left(F_{2}+N_{2}\right) A_{\tau}-A_{\tau}^{\prime}\left(F_{2}^{\prime}+N_{2}^{\prime}\right) \\
-G_{2} A_{\tau} A-A^{\prime} A_{\tau}^{\prime} G_{2}^{\prime}-H_{2}-H_{2}^{\prime} \\
\Phi_{3}=G_{2}-A^{\prime} F_{3}^{\prime}-A_{\tau}^{\prime}\left(N_{3}^{\prime}+F_{3}^{\prime}\right)-A^{\prime} A_{\tau}^{\prime} G_{3}^{\prime}-H_{3}^{\prime} \\
\Phi_{4}=H_{2}-A^{\prime} F_{4}^{\prime}-A_{\tau}^{\prime}\left(N_{4}^{\prime}+F_{4}^{\prime}\right)-A^{\prime} A_{\tau}^{\prime} G_{4}^{\prime}-H_{4}^{\prime} \\
\Phi_{5}=-\bar{\tau} F_{2}-\bar{\tau} N_{2}-A^{\prime} F_{5}^{\prime}-A_{\tau}^{\prime}\left(N_{5}^{\prime}+F_{5}^{\prime}\right) \\
-A^{\prime} A_{\tau}^{\prime} G_{5}^{\prime}-H_{5}^{\prime} \\
\Phi_{6}=N_{2} A_{\tau}-G_{2} A_{\tau} A_{\tau}-A^{\prime} F_{6}^{\prime} \\
-A_{\tau}^{\prime}\left(N_{6}^{\prime}+F_{6}^{\prime}\right)-A^{\prime} A_{\tau}^{\prime} G_{6}^{\prime}-H_{6}^{\prime} \\
\Phi_{7}=N_{6} A_{\tau}-G_{6} A_{\tau} A_{\tau}+A_{\tau}^{\prime} N_{6}^{\prime}-A_{\tau}^{\prime} A_{\tau}^{\prime} G_{6}^{\prime}-U
\end{gathered}
$$

Prova: Semelhantemente, a escolha

$$
\begin{gathered}
\mathscr{A}=A+M A_{\tau}, \mathscr{B}=\left[\begin{array}{cc}
\bar{\tau} M & (\mathbf{I}-M) A_{\tau}
\end{array}\right], \\
\mathscr{C}=\left[\begin{array}{c}
A_{\tau} A \\
\mathbf{I}
\end{array}\right], \mathscr{D}=\left[\begin{array}{cc}
\mathbf{0} & A_{\tau} A_{\tau} \\
\mathbf{0} & \mathbf{0}
\end{array}\right]
\end{gathered}
$$

substituída no Lema 1 resulta em (Zhang et al., 2001) em condições suficientes para a estabilidade do sistema (1)-(2) para $0 \leq \tau \leq \bar{\tau}$ com $M$ sendo considerada uma variável matricial livre. A mesma escolha aplicada às condições do Lema 2, com as mudanças de variáveis $N_{i}=F_{i}(M-\mathbf{I})$, $i=1, \ldots, 6$ produz as LMIs do Lema 5. Assim como em Zhang et al. (2001), é possível fazer essa mudança pois $M$ e $N_{i}, i=1, \ldots, 6$ são variáveis livres.

\subsubsection{Funcionais de Lyapunov-Krasovskii}

É importante destacar que as condições obtidas a partir do teorema do ganho pequeno escalonado, com as escolhas (9), (12) e (15), apresentadas em Zhang et al. (2001), quando aplicadas ao Lema 1, correspondem às condições de estabilidade de sistemas com atrasos obtidas a partir de funcionais de Lyapunov-Krasovskii, como publicado na literatura (Verriest et al., 1993), (Li e de Souza, 1996), (Park, 1999).

A condição independente do atraso associada à escolha (9) é obtida em Verriest et al. (1993) a partir do funcional de Lyapunov-Krasovskii dado por

$$
V\left(x_{t}\right)=x(t)^{\prime} P x(t)+\int_{-\tau}^{0} x(t+\theta)^{\prime} Q x(t+\theta) d \theta
$$




$$
\begin{aligned}
& {\left[\begin{array}{cccc}
F_{1}+F_{1}^{\prime} & -F_{1} A-G_{1}+F_{2}^{\prime}+P & G_{1}+F_{3}^{\prime} & -F_{1} A_{\tau}+F_{4}^{\prime} \\
\star & -F_{2} A-A^{\prime} F_{2}^{\prime}-G_{2}-G_{2}^{\prime} & G_{2}-A^{\prime} F_{3}^{\prime}-G_{3}^{\prime} & -F_{2} A_{\tau}-A^{\prime} F_{4}^{\prime}-G_{4}^{\prime} \\
\star & \star & Q+G_{3}+G_{3}^{\prime} & -F_{3} A_{\tau}+G_{4}^{\prime} \\
\star & \star & \star & -Q-F_{4} A_{\tau}-A_{\tau}^{\prime} F_{4}^{\prime}
\end{array}\right]<0} \\
& {\left[\begin{array}{cccccc}
F_{1}+F_{1}^{\prime} & \Psi_{1} & G_{1}+F_{3}^{\prime} & H_{1}+F_{4}^{\prime} & -\bar{\tau} F_{1} A_{\tau}+F_{5}^{\prime} & -\bar{\tau} F_{1} A_{\tau}+F_{6}^{\prime} \\
\star & \Psi_{2} & \Psi_{3} & \Psi_{4} & \Psi_{5} & \Psi_{6} \\
\star & \star & G_{3}+G_{3}^{\prime}+V & H_{3}+G_{4}^{\prime} & -\bar{\tau} F_{3} A_{\tau}+G_{5}^{\prime} & -\bar{\tau} F_{3} A_{\tau}+G_{6}^{\prime} \\
\star & \star & \star & H_{4}+H_{4}^{\prime}+U & -\bar{\tau} F_{4} A_{\tau}+H_{5}^{\prime} & -\bar{\tau} F_{4} A_{\tau}+H_{6}^{\prime} \\
\star & \star & \star & \star & -\bar{\tau} F_{5} A_{\tau}-\bar{\tau} A_{\tau}^{\prime} F_{5}^{\prime}-V & -\bar{\tau} F_{5} A_{\tau}-\bar{\tau} A_{\tau}^{\prime} F_{6}^{\prime} \\
\star & \star & \star & \star & \star & -\bar{\tau} F_{6} A_{\tau}-\bar{\tau} A_{\tau}^{\prime} F_{6}^{\prime}-U
\end{array}\right]<0} \\
& {\left[\begin{array}{cccccc}
F_{1}+F_{1}^{\prime} & \Phi_{1} & G_{1}+F_{3}^{\prime} & H_{1}+F_{4}^{\prime} & -\bar{\tau} F_{1}-\bar{\tau} N_{1}+F_{5}^{\prime} & N_{1} A_{\tau}-G_{1} A_{\tau} A_{\tau}+F_{6}^{\prime} \\
\star & \Phi_{2} & \Phi_{3} & \Phi_{4} & \Phi_{5} & \Phi_{6} \\
\star & \star & G_{3}+G_{3}^{\prime}+V & H_{3}+G_{4}^{\prime} & -\bar{\tau} F_{3}-\bar{\tau} N_{3}+G_{5}^{\prime} & N_{3} A_{\tau}-G_{3} A_{\tau} A_{\tau}+G_{6}^{\prime} \\
\star & \star & \star & H_{4}+H_{4}^{\prime}+U & -\bar{\tau} F_{4}-\bar{\tau} N_{4}+H_{5}^{\prime} & N_{4} A_{\tau}-G_{4} A_{\tau} A_{\tau}+H_{6}^{\prime} \\
\star & \star & \star & \star & \star \bar{\tau} F_{5}-\bar{\tau} N_{5}-\bar{\tau} F_{5}^{\prime}-\bar{\tau} N_{5}^{\prime}-V & N_{5} A_{\tau}-G_{5} A_{\tau} A_{\tau}-\bar{\tau}\left(F_{6}^{\prime}+N_{6}^{\prime}\right) \\
\star & \star & \star & \star & \star & \Phi_{7}
\end{array}\right]<0}
\end{aligned}
$$

sendo que $x_{t}$ denota $x(t)$ para $t \in[t-\tau, t]$. A condição dependente do atraso correspondente à escolha (12) foi proposta em Li e de Souza (1996) e pode ser obtida com o funcional

$$
\begin{aligned}
V\left(x_{t}\right)=x(t)^{\prime} P x(t) & +\int_{-\tau}^{0}\left[\int_{t+\theta}^{t} x(\beta)^{\prime} A^{\prime} Q_{1} A x(\beta) d \beta\right. \\
& \left.+\int_{t+\theta-\tau}^{t} x(\beta)^{\prime} A_{\tau}^{\prime} Q_{2} A_{\tau} x(\beta) d \beta\right] d \theta
\end{aligned}
$$

e, finalmente, a condição dependente do atraso correspondente à escolha (15), proposta em Park (1999), é obtida com o funcional

$$
\begin{array}{r}
V\left(x_{t}\right)=x(t)^{\prime} P x(t)+\int_{-\tau}^{0} \int_{t+\theta}^{t} \dot{x}(\beta)^{\prime} A_{\tau}^{\prime} Q_{1} A_{\tau} \dot{x}(\beta) d \beta d \theta \\
+\int_{t-\tau}^{t} x(\theta)^{\prime} Q_{2} x(\theta) d \theta
\end{array}
$$

\subsection{Análise de Sistemas Incertos}

Considere que as matrizes $A$ e $A_{\tau}$ não são precisamente conhecidas, mas pertencem a um domínio politópico de incerteza $\mathscr{P}$ dado por

$$
\begin{array}{r}
\mathscr{P}=\left\{\left(A, A_{\tau}\right)(\xi):\left(A, A_{\tau}\right)(\xi)=\sum_{j=1}^{N} \xi_{j}\left(A, A_{\tau}\right)_{j}\right. \\
\left.\sum_{j=1}^{N} \xi_{j}=1 ; \xi_{j} \geq 0\right\}
\end{array}
$$

Qualquer par de matrizes no conjunto $\mathscr{P}$ pode ser escrito como uma combinação convexa dos vértices $\left(A, A_{\tau}\right)_{j}$ do politopo. Nas LMIs seguintes, as matrizes $A_{j}$ e $A_{\tau j}$, mesmo aparecendo separadamente, correspondem ao vértice $\left(A, A_{\tau}\right)_{j}$ de $\mathscr{P}, j=1, \ldots, N$.

$\mathrm{Na}$ literatura, as condições de estabilidade robusta para sistemas com atraso descritos por (1)-(2) $\operatorname{com}\left(A, A_{\tau}\right) \in$ $\mathscr{P}$ geralmente empregam estabilidade quadrática (ou seja, $P$ e $Q$ são consideradas matrizes constantes nos Lemas 1 e 2). A estabilidade quadrática pode ser verificada para todo $\left(A, A_{\tau}\right) \in \mathscr{P}$ testando as condições (por exemplo a condição do Lema 1) nos vértices $\left(A, A_{\tau}\right)_{j}, j=1, \ldots, N$ do politopo $\mathscr{P}$. Resultados menos conservadores que contêm a estabilidade quadrática como um caso particular podem ser obtidos considerando matrizes dependentes de parâmetro $P(\xi)=P(\xi)^{\prime}>0$ e $Q(\xi)=Q(\xi)^{\prime}>0$ dadas por

$$
P(\xi)=\sum_{j=1}^{N} \xi_{j} P_{j} ; Q(\xi)=\sum_{j=1}^{N} \xi_{j} Q_{j} ; \sum_{j=1}^{N} \xi_{j}=1 ; \xi_{j} \geq 0
$$

no Lema 1.

Empregando a escolha dada por (9) para $(\mathscr{A}, \mathscr{B}, \mathscr{C}, \mathscr{D})$, nos vértices de $\mathscr{P}$, a estabilidade robusta do sistema (1)-(2) com $\left(A, A_{\tau}\right) \in \mathscr{P}$ é garantida pelos seguintes lemas.

Lema $6 \mathrm{O}$ sistema (1)-(2) $\operatorname{com}\left(A, A_{\tau}\right) \in \mathscr{P}$ dado por (16) é robustamente estável independentemente da magnitude do atraso se existirem $P_{j}=P_{j}^{\prime}>0$ e $Q_{j}=Q_{j}^{\prime}, j=1, \ldots, N$, matrizes do $\mathbb{R}^{n \times n}$, tais que

$$
\begin{aligned}
& W_{j}=\left[\begin{array}{ccc}
\mathscr{A}_{j}^{\prime} P_{j}+P_{j} \mathscr{A}_{j} & P_{j} \mathscr{B}_{j} & \mathscr{C}_{j}^{\prime} Q_{j} \\
\star & -Q_{j} & \mathscr{D}_{j}^{\prime} Q_{j} \\
\star & \star & -Q_{j}
\end{array}\right]<0 ; \\
& j=1, \ldots, N
\end{aligned}
$$




$$
\begin{gathered}
W_{j k}=\left[\begin{array}{ccc}
\Xi & P_{k} \mathscr{B}_{j}+P_{j} \mathscr{B}_{k} & \mathscr{C}_{j}^{\prime} Q_{k}+\mathscr{C}_{k}^{\prime} Q_{j} \\
\star & -Q_{j}-Q_{k} & \mathscr{D}_{j}^{\prime} Q_{k}+\mathscr{D}_{k}^{\prime} Q_{j} \\
\star & \star & -Q_{j}-Q_{k}
\end{array}\right]<0 ; \\
\Xi=\mathscr{A}_{j}^{\prime} P_{k}+P_{k} \mathscr{A}_{j}+\mathscr{A}_{k}^{\prime} P_{j}+P_{j} \mathscr{A}_{k} \\
j=1, \ldots, N-1 ; k=j+1, \ldots, N \\
\mathscr{A}_{j}=A_{j}, \mathscr{B}_{j}=A_{\tau j}, \\
\mathscr{C}_{j}=\mathbf{I}, \mathscr{D}_{j}=\mathbf{0} ; j=1, \ldots, N
\end{gathered}
$$

Prova: Com a escolha $\mathscr{A}_{j}, \mathscr{B}_{j}, \mathscr{C}_{j}, \mathscr{D}_{j}$ dada por $(20), P(\xi)=$ $P(\xi)^{\prime}>0$ e $Q(\xi)=Q(\xi)^{\prime}>0$ dadas por (17), substituindose nas condições do Lema 6 tem-se

$$
\begin{array}{r}
W(\xi)=\left[\begin{array}{cc}
\mathscr{A}(\xi)^{\prime} P(\xi)+P(\xi) \mathscr{A}(\xi) & P(\xi) \mathscr{B}(\xi) \\
\star & -Q(\xi) \\
\star & \star \\
\mathscr{C}(\xi)^{\prime} Q(\xi) \\
\mathscr{D}(\xi)^{\prime} Q(\xi) \\
-Q(\xi)
\end{array}\right] \\
=\sum_{j=1}^{N} \xi_{j}^{2} W_{j}+\sum_{j=1}^{N-1} \sum_{k=j+1}^{N} \xi_{j} \xi_{k} W_{j k}<0
\end{array}
$$

o que assegura que o sistema incerto (1)-(2) $\operatorname{com}\left(A, A_{\tau}\right) \in \mathscr{P}$ dado por (16) é robustamente estável independentemente do atraso.

Usando os graus de liberdade extras proporcionados pelas matrizes $\mathscr{X}$ no Lema 2, o seguinte resultado é proposto.

Lema $7 \mathrm{O}$ sistema (1)-(2) com $\left(A, A_{\tau}\right) \in \mathscr{P}$ dado por (16) é robustamente estável independentemente do atraso se existirem $P_{j}=P_{j}^{\prime}>0, Q_{j}=Q_{j}^{\prime}>0$ e $\mathscr{X}_{j}, j=1, \ldots, N$, matrizes do $\mathbb{R}^{n \times n}$, tais que

$$
\begin{gathered}
\tilde{Q}_{j}+\mathscr{X}_{j} \tilde{B}_{j}+\tilde{B}_{j}^{\prime} \mathscr{X}_{j}^{\prime}<0 ; j=1, \ldots, N \\
\tilde{Q}_{j}+\tilde{Q}_{k}+\mathscr{X}_{j} \tilde{B}_{k}+\mathscr{X}_{k} \tilde{B}_{j}+\tilde{B}_{j}^{\prime} \mathscr{X}_{k}^{\prime}+\tilde{B}_{k}^{\prime} \mathscr{X}_{j}^{\prime}<0 ; \\
j=1, \ldots, N-1 ; k=j+1, \ldots, N
\end{gathered}
$$

$\operatorname{com} \mathscr{A}_{j}, \mathscr{B}_{j}, \mathscr{C}_{j}, \mathscr{D}_{j}$ dadas em (20) e

$$
\tilde{Q}_{j}=\left[\begin{array}{cccc}
\mathbf{0} & P_{j} & \mathbf{0} & \mathbf{0} \\
\star & \mathbf{0} & \mathbf{0} & \mathbf{0} \\
\star & \star & Q_{j} & \mathbf{0} \\
\star & \star & \star & -Q_{j}
\end{array}\right] ; j=1, \ldots, N
$$

Prova: A prova é bastante semelhante à prova do Lema 6 . As LMIs do Lema 7 asseguram que existem $P(\xi)=P(\xi)^{\prime}>0$ e $Q(\xi)=Q(\xi)^{\prime}>0$ como em (17) e $\mathscr{X}(\xi)$ dada por

$$
\mathscr{X}(\xi)=\sum_{j=j}^{N} \xi_{j} \mathscr{X}_{j}, \sum_{j=1}^{N} \xi_{j}=1, \xi_{j} \geq 0
$$

tais que

$$
\tilde{Q}(\xi)+\mathscr{X}(\xi) \tilde{B}(\xi)+\tilde{B}(\xi)^{\prime} \mathscr{X}(\xi)^{\prime}<0
$$

que, de acordo com o Lema 2, é equivalente a (21).

A escolha dada por (12) para $(\mathscr{A}, \mathscr{B}, \mathscr{C}, \mathscr{D})$, nos vértices de $\mathscr{P}$, a estabilidade robusta do sistema (1)-(2) $\operatorname{com}\left(A, A_{\tau}\right) \in$ $\mathscr{P}$ é garantida pelos lemas abaixo.

Lema 8 O sistema (1)-(2) com $\left(A, A_{\tau}\right) \in \mathscr{P}$ dado por (16) é robustamente estável para $0 \leq \tau \leq \bar{\tau}$ se existirem $P_{j}=P_{j}^{\prime}>0$, $V_{j}=V_{j}^{\prime}>0$ e $U_{j}=U_{j}^{\prime}>0, j=1, \ldots, N$, matrizes do $\mathbb{R}^{n \times n}$ com

$$
Q_{j}=Q_{j}^{\prime}=\operatorname{diag}\left\{V_{j}, U_{j}\right\}>0,
$$

tais que (18)-(19) são satisfeitas e

$$
\begin{gathered}
\mathscr{A}_{j}=A_{j}+A_{\tau j}, \mathscr{B}_{j}=\left[\begin{array}{ll}
\bar{\tau} A_{\tau j} & \bar{\tau} A_{\tau j}
\end{array}\right], \\
\mathscr{C}_{j}=\left[\begin{array}{c}
A_{j} \\
A_{\tau j}
\end{array}\right], \mathscr{D}_{j}=\mathbf{0} ; j=1, \ldots, N
\end{gathered}
$$

Prova: Com a escolha $\mathscr{A}_{j}, \mathscr{B}_{j}, \mathscr{C}_{j}, \mathscr{D}_{j}$ dada por (28), $P(\xi)=$ $P(\xi)^{\prime}>0$ e $Q(\xi)=Q(\xi)^{\prime}>0$ dadas por (17), substituindose nas condições do Lema 8 tem-se (21), o que assegura que o sistema incerto (1)-(2) com $\left(A, A_{\tau}\right) \in \mathscr{P}$ dado por (16) é robustamente estável para $0 \leq \tau \leq \bar{\tau}$.

Resultados menos conservadores são obtidos usando as variáveis matriciais extras introduzidas pelo Lema 2.

Lema 9 O sistema (1)-(2) com $\left(A, A_{\tau}\right) \in \mathscr{P}$ dado por (16) é robustamente estável para $0 \leq \tau \leq \bar{\tau}$ se existirem $P_{j}=P_{j}^{\prime}>0$, $V_{j}=V_{j}^{\prime}>0, U_{j}=U_{j}^{\prime}>0$ e $\mathscr{X}_{j}, j=1, \ldots, N$, matrizes do $\mathbb{R}^{n \times n}$, tais que (22)-(23) são satisfeitas com $\mathscr{A}_{j}, \mathscr{B}_{j}, \mathscr{C}_{j}, \mathscr{D}_{j}$ dadas em (28), $Q_{j}=Q_{j}^{\prime}>0$ dada por (27) e $\tilde{Q}_{j}$ como em (24).

Prova: A prova é bastante semelhante à prova do Lema 8 . As LMIs do Lema 9 asseguram que existem $P(\xi)=P(\xi)^{\prime}>0$ e $Q(\xi)=Q(\xi)^{\prime}>0$ como em (17) e $\mathscr{X}(\xi)$ dada por (25) tais que (26) é válida, o que, de acordo com o Lema 2, garante que (21) também é verificada.

Como feito para sistemas precisamente conhecidos, a escolha de $(\mathscr{A}, \mathscr{B}, \mathscr{C}, \mathscr{D})$ dada por (15) nos vértices do politopo $\mathscr{P}$ resulta em condições de estabilidade robusta dependentes do atraso para o sistema (1)-(2). Como existem produtos de matrizes tais como $A_{\tau} A$ e $A_{\tau} A_{\tau}$, as LMIs devem considerar três índices: $j, k \mathrm{e} \ell$.

Lema $10 \mathrm{O}$ sistema (1)-(2) $\operatorname{com}\left(A, A_{\tau}\right) \in \mathscr{P}$ dado por (16) é robustamente estável $0 \leq \tau \leq \bar{\tau}$ se existirem $P_{j}=P_{j}^{\prime}>0$, $V_{j}=V_{j}^{\prime}>0, U_{j}=U_{j}^{\prime}>0$ e $N_{j}, j=1, \ldots, N$, matrizes do $\mathbb{R}^{n \times n}$, tais que as LMIs (29), (30) e (31) sejam satisfeitas. 


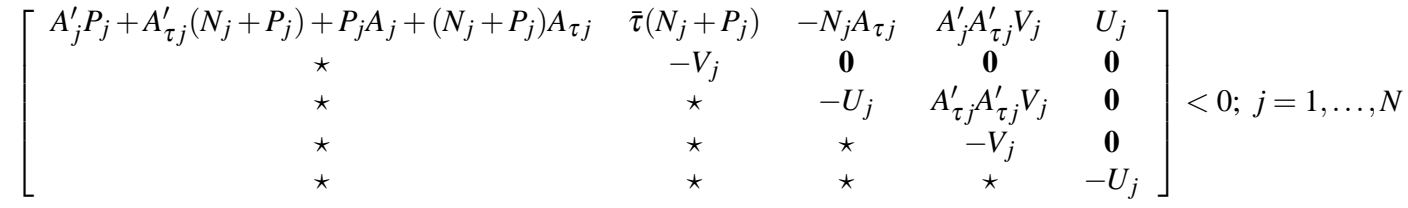

$$
\begin{aligned}
& {\left[\begin{array}{cc}
\Gamma_{1} & 2 \bar{\tau}\left(N_{j}+P_{j}\right)+\bar{\tau}\left(N_{k}+P_{k}\right) \\
\star & -2 V_{j}-V_{k} \\
\star & \star \\
\star & \star \\
\star & \star
\end{array}\right.} \\
& \begin{array}{cc}
\Gamma_{2} & A_{k}^{\prime} A_{\tau j}^{\prime} V_{j}+A_{j}^{\prime} A_{d k}^{\prime} V_{j}+A_{j}^{\prime} A_{\tau j}^{\prime} V_{k} \\
\mathbf{0} & \mathbf{0} \\
-2 U_{j}-U_{k} & A_{d k}^{\prime} A_{\tau j}^{\prime} V_{j}+A_{\tau j}^{\prime} A_{d k}^{\prime} V_{j}+A_{\tau j}^{\prime} A_{\tau j}^{\prime} V_{k} \\
\star & -2 V_{j}-V_{k} \\
\star & \star
\end{array} \\
& \left.\begin{array}{c}
2 U_{j}+U_{k} \\
\mathbf{0} \\
\mathbf{0} \\
\mathbf{0} \\
-2 U_{j}-U_{k}
\end{array}\right]<0 \\
& j=1, \ldots, N ; k=1, \ldots, N ; k \neq j \\
& \Gamma_{1}=A_{j}^{\prime} P_{j}+A_{\tau j}^{\prime}\left(N_{j}+P_{j}\right)+P_{j} A_{j}+\left(N_{j}^{\prime}+P_{j}\right) A_{\tau j}+A_{j}^{\prime} P_{k}+A_{\tau j}^{\prime}\left(N_{k}+P_{k}\right) \\
& +P_{j} A_{k}+\left(N_{j}^{\prime}+P_{j}\right) A_{d k}+A_{k}^{\prime} P_{j}+A_{d k}^{\prime}\left(N_{j}+P_{j}\right)+P_{k} A_{j}+\left(N_{j}^{\prime}+P_{j}\right) A_{\tau j} \\
& \Gamma_{2}=-N_{j} A_{\tau j}-N_{k} A_{\tau j}-N_{j} A_{d k} \\
& {\left[\begin{array}{cc}
\Xi_{1} & 2\left(\bar{\tau}\left(N_{j}+P_{j}\right)+\bar{\tau}\left(N_{k}+P_{k}\right)+\bar{\tau}\left(N_{\ell}+P_{\ell}\right)\right) \\
\star & -2\left(V_{j}+V_{k}+V_{\ell}\right) \\
\star & \star \\
\star & \star \\
\star & \star
\end{array}\right.} \\
& \left.\begin{array}{ccc}
\Xi_{2} & \Xi_{3} & 2\left(U_{j}+U_{k}+U_{\ell}\right) \\
\mathbf{0} & \mathbf{0} & \mathbf{0} \\
-2\left(U_{j}+U_{k}+U_{\ell}\right) & \Xi_{4} & \mathbf{0} \\
\star & -2\left(V_{j}+V_{k}+V_{\ell}\right) & \mathbf{0} \\
\star & \star & -2\left(U_{j}+U_{k}+U_{\ell}\right)
\end{array}\right]<0 ; \\
& j=1, \ldots, N-2 ; k=j+1, \ldots, N-1 ; \ell=k+1, \ldots, N \\
& \Xi_{1}=A_{j}^{\prime} P_{k}+A_{k}^{\prime} P_{j}+A_{j}^{\prime} P_{\ell}+A_{\ell}^{\prime} P_{j}+A_{k}^{\prime} P_{\ell}+A_{\ell}^{\prime} P_{k}+A_{\tau j}^{\prime}\left(N_{k}+P_{k}\right)+A_{d k}^{\prime}\left(N_{j}+P_{j}\right)+A_{\tau j}^{\prime}\left(N_{\ell}+P_{\ell}\right) \\
& +A_{d \ell}^{\prime}\left(N_{j}+P_{j}\right)+A_{d k}^{\prime}\left(N_{\ell}+P_{\ell}\right)+A_{d \ell}^{\prime}\left(N_{k}+P_{k}\right)+P_{j} A_{k}+P_{k} A_{j}+P_{j} A_{\ell}+P_{\ell} A_{j}+P_{k} A_{\ell}+P_{\ell} A_{k} \\
& +\left(N_{j}^{\prime}+P_{j}\right) A_{d k}+\left(N_{k}^{\prime}+P_{k}\right) A_{\tau j}+\left(N_{j}^{\prime}+P_{j}\right) A_{d \ell}+\left(N_{\ell}^{\prime}+P_{\ell}\right) A_{\tau j}+\left(N_{k}^{\prime}+P_{k}\right) A_{d \ell}+\left(N_{\ell}^{\prime}+P_{\ell}\right) A_{d k} \\
& \Xi_{2}=-N_{j} A_{d k}-N_{k} A_{\tau j}-N_{j} A_{d \ell}-N_{\ell} A_{\tau j}-N_{k} A_{d \ell}-N_{\ell} A_{d k} \\
& \Xi_{3}=A_{k}^{\prime} A_{\tau j}^{\prime} V_{\ell}+A_{k}^{\prime} A_{d \ell}^{\prime} V_{j}+A_{j}^{\prime} A_{d k}^{\prime} V_{\ell}+A_{\ell}^{\prime} A_{d k}^{\prime} V_{j}+A_{j}^{\prime} A_{d \ell}^{\prime} V_{k}+A_{\ell}^{\prime} A_{\tau j}^{\prime} V_{k} \\
& \Xi_{4}=A_{d k}^{\prime} A_{\tau j}^{\prime} V_{\ell}+A_{d k}^{\prime} A_{d \ell}^{\prime} V_{j}+A_{\tau j}^{\prime} A_{d k}^{\prime} V_{\ell}+A_{d \ell}^{\prime} A_{d k}^{\prime} V_{j}+A_{\tau j}^{\prime} A_{d \ell}^{\prime} V_{k}+A_{d \ell}^{\prime} A_{\tau j}^{\prime} V_{k}
\end{aligned}
$$

Prova: A prova é semelhante às provas dos Lemas 8 e 9. As LMIs (29), (30) e (31) são suficientes para garantir que $P(\xi)=P(\xi)^{\prime}>0, Q(\xi)=Q(\xi)^{\prime}>0$ dadas por (17) com $Q_{j}$ definida em (27) verifiquem as condições do Lema 1 para todo $\left(A, A_{\tau}\right) \in \mathscr{P}$. Note que foi utilizada a mudança de variáveis $N_{j}=P_{j}(M-\mathbf{I}), j=1, \ldots, N$.

Usando as variáveis extras do Lema 2, resultados menos conservadores são obtidos.

Lema $11 \mathrm{O}$ sistema (1)-(2) $\operatorname{com}\left(A, A_{\tau}\right) \in \mathscr{P}$ dado por (16) é robustamente estável para $0 \leq \tau \leq \bar{\tau}$ se existirem $P_{j}=P_{j}^{\prime}>$ $0, V_{j}=V_{j}^{\prime}>0, U_{j}=U_{j}^{\prime}>0$ e $N_{1 j}, F_{1 j}, G_{1 j}, H_{1 j}, \ldots, N_{6 j}, F_{6 j}$, $G_{6 j}, H_{6 j}, j=1, \ldots, N$, matrizes do $\mathbb{R}^{n \times n}$, tais que as LMIs (32), (33) e (34) sejam satisfeitas.

Prova: Semelhante à prova do Lema 10. As LMIs (32), (33) e (34) são suficientes para garantir que $P(\xi)=P(\xi)^{\prime}>0$,
$Q(\xi)=Q(\xi)^{\prime}>0$ dadas por (17) com $Q_{j}$ definida em (27) e $\mathscr{X}(\xi)$ dada por (25) com $\mathscr{X}_{j}$ particionada como em (13) verificam as condições do Lema 5 para todo $\left(A, A_{\tau}\right) \in \mathscr{P}$. Foi realizada a mudança de variáveis $N_{\ell j}=F_{\ell j}(M-\mathbf{I}), \ell=$ $1, \ldots, 6, j=1, \ldots, N$, similar à do Lema 10.

Um primeiro comentário sobre os resultados desta seção é que, embora as LMIs dependentes de parâmetro (21) e (26) sejam equivalentes, as variáveis matriciais extras $\mathscr{X}_{j}$ fazem com que as condições suficientes dos Lemas 7, 9 e 11 (obtidos pelo Lema de Finsler) sejam menos conservadoras que as condições suficientes dos correspondentes Lemas 6, 8 e 10 (obtidas diretamente pela exploração da positividade do conjunto de parâmetros incertos) para a avaliação das condições de estabilidade robusta dos sistemas investigados.

Outro ponto a ressaltar é que as condições dos Lemas 7, 9 e 11 poderiam ser usadas com as matrizes extras (partições 


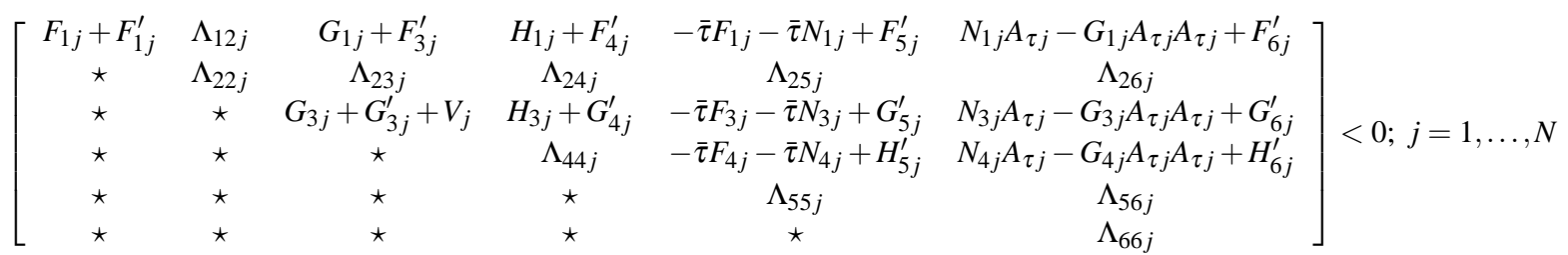

com

$$
\begin{gathered}
\Lambda_{12 j}=-F_{1 j} A_{j}-\left(F_{1 j}+N_{1 j}\right) A_{\tau j}-G_{1 j} A_{\tau j} A_{j}-H_{1 j}+F_{2 j}^{\prime} \\
\Lambda_{22 j}=-F_{2 j} A_{j}-A_{j}^{\prime} F_{2 j}^{\prime}-\left(F_{2 j}+N_{2 j}\right) A_{\tau j}-A_{\tau j}^{\prime}\left(F_{2 j}^{\prime}+N_{2 j}^{\prime}\right)-G_{2 j} A_{\tau j} A_{j}-A_{j}^{\prime} A_{\tau j}^{\prime} G_{2 j}^{\prime}-H_{3 j}-H_{3 j}^{\prime} \\
\Lambda_{23 j}=G_{2 j}-A_{j}^{\prime} F_{3 j}^{\prime}-A_{\tau j}^{\prime}\left(N_{3 j}^{\prime}+F_{3 j}^{\prime}\right)-A_{j}^{\prime} A_{\tau j}^{\prime} G_{3 j}^{\prime}-H_{3 j}^{\prime} ; \Lambda_{24 j}=H_{3 j}-A_{j}^{\prime} F_{4 j}^{\prime}-A_{\tau j}^{\prime}\left(N_{4 j}^{\prime}+G_{4 j}^{\prime}\right)-A_{j}^{\prime} A_{\tau j}^{\prime} G_{4 j}^{\prime}-H_{4 j}^{\prime} \\
\Lambda_{25 j}=-\bar{\tau} F_{2 j}-\bar{\tau} N_{2 j}-A_{j}^{\prime} F_{5 j}^{\prime}-A_{\tau j}^{\prime}\left(N_{5 j}^{\prime}+F_{5 j}^{\prime}\right)-A_{j}^{\prime} A_{\tau j}^{\prime} G_{5 j}^{\prime}-H_{5 j}^{\prime} \\
\Lambda_{26 j}=-N_{2 j} A_{\tau j}-G_{2 j} A_{\tau j} A_{\tau j}-A_{j}^{\prime} F_{6 j}^{\prime}-A_{\tau j}^{\prime}\left(N_{6 j}^{\prime}+F_{6 j}^{\prime}\right)-A_{j}^{\prime} A_{\tau j}^{\prime} G_{6 j}^{\prime}-H_{6 j}^{\prime} \\
\Lambda_{44 j}=H_{4 j}+H_{4 j}^{\prime}+U_{j} ; \Lambda_{55 j}=-\bar{\tau} F_{5 j}-\bar{\tau} N_{5 j}-\bar{\tau} F_{5 j}^{\prime}-\bar{\tau} N_{5 j}^{\prime}-V_{j} ; \Lambda_{56 j}=N_{5 j} A_{\tau j}-G_{5 j} A_{\tau j} A_{\tau j}-\bar{\tau}\left(F_{6 j}^{\prime}+N_{6 j}^{\prime}\right) \\
\Lambda_{66 j}=-N_{6 j} A_{\tau j}-G_{6 j} A_{\tau j} A_{\tau j}+A_{\tau j}^{\prime} N_{6 j}^{\prime}-A_{\tau j}^{\prime} A_{\tau j}^{\prime} G_{6 j}^{\prime}-U_{j}
\end{gathered}
$$

da matriz $\mathscr{X}$ ) fixas, resultando em condições mais simples e de menor complexidade, porém mais conservadoras.

\subsection{Complexidade das Condições LMIs}

As condições fornecidas nos lemas 3 a 11 são convexas, definidas como testes de factibilidade de LMIs. Podem ser resolvidas em tempo polinomial por algoritmos de pontos interiores, com esforço computational proporcional a $K^{3} L$, sendo $K$ o número de variáveis escalares e $L$ o número de linhas de LMIs (Boyd et al., 1994), (Gahinet et al., 1995). Outros programas resolvedores de LMIs, como por exemplo o SeDuMi (Sturm, 1999), podem exigir esforços computacionais diferentes. A Tabela 1 dá os valores de $K$ e $L$ para cada um lemas propostos.

\section{EXEMPLOS NUMÉRICOS}

Considere o sistema incerto apresentado em Peres et al. (2003) com vértices dados por

$$
\begin{aligned}
& \left(A, A_{\tau}\right)_{1}=\left(\left[\begin{array}{cc}
-1.3451 & 0.6510 \\
0.6135 & -0.3007
\end{array}\right],\left[\begin{array}{cc}
0.0025 & -0.7350 \\
0.0859 & -0.0086
\end{array}\right]\right) \\
& \left(A, A_{\tau}\right)_{2}=\left(\left[\begin{array}{ll}
-0.1849 & 0.1202 \\
-0.9822 & 0.1787
\end{array}\right],\left[\begin{array}{cc}
-0.3219 & 0.1123 \\
0.4372 & -0.1571
\end{array}\right]\right)
\end{aligned}
$$

O atraso máximo $\bar{\tau}$ para o qual a estabilidade robusta é assegurada foi calculado testando as condições dos Lemas 8 (L8), 9 (L9), 10 (L10) e 11 (L11), resultando nos seguintes valores: $\bar{\tau}_{L 8}=0.249, \bar{\tau}_{L 9}=0.712, \bar{\tau}_{L 10}=1.699$ e $\bar{\tau}_{L 11}=$ 2.191. Em Peres et al. (2003) o melhor resultado obtido foi $\bar{\tau}=1.480$, aqui superado pela avaliação obtida com o Lema 11 .
Considere o seguinte modelo para a dinâmica de um processo de fresagem, no qual algumas incertezas foram inseridas Zhang et al. (2002)

$$
\dot{x}=A(k, \rho) x(t)+A_{\tau}(k) x(t-\tau)
$$

com

$$
\begin{gathered}
A(k, \rho, \beta)=\left[\begin{array}{cccc}
0 & 0 & 1 & 0 \\
0 & 0 & 0 & 1 \\
a_{31} & 9.5+\rho & 0 & 0 \\
4.75+\frac{\rho}{2} & -14.75-\frac{\rho}{2} & 0 & -0.25
\end{array}\right] \\
A_{\tau}(k)=\left[\begin{array}{llll}
0 & 0 & k & 0
\end{array}\right]^{\prime}\left[\begin{array}{cccc}
1 & 0 & 0 & 0
\end{array}\right]
\end{gathered}
$$

O elemento $a_{31}$ na matriz dinâmica $A$ é dado por $a_{31}=$ $-(9.5+\rho+k)$, sendo que $k$ representa a rigidez de corte, e $0 \leq \rho \leq 1$. O sistema precisamente conhecido apresentado em Zhang et al. (2001) é obtido com o valor $\rho=0.5$. Primeiramente, considere $k=0.1$, definindo assim um politopo com dois vértices ( $V_{1}$ para $\rho=0$ e $V_{2}$ para $\rho=1$ ). Para este caso o Lema 10 assegura a estabilidade robusta dependente do atraso para o sistema incerto até o valor $\bar{\tau}_{L 10}=1 \times 10^{16}$, o que pode ser um indicativo de que o sistema é estável independentemente do atraso. Os Lemas $8 \mathrm{e}$ 9 garantem a estabilidade robusta dependente do atraso para $\bar{\tau}_{L 8}=0.624$ e $\bar{\tau}_{L 9}=0.231$, respectivamente.

Considerando o caso em que $k=0.5$, tem-se outro politopo com dois vértices. As condições dos Lemas 8 a 11 (L8 a L11) asseguram a estabilidade robusta dependente do atraso para os seguintes valores máximos de atraso: $\bar{\tau}_{L 8}=0.124$, $\bar{\tau}_{L 9}=0.231, \bar{\tau}_{L 10}=0.244, \bar{\tau}_{L 11}=0.320$.

As condições propostas para análise de sistemas incertos nos Lemas 6 e 7 foram testadas para o seguinte sistema incerto de ordem três e com dois vértices randomicamente gerados 
com

$$
\left[\begin{array}{ccc}
\Lambda_{11 j k} & \cdots & \Lambda_{16 j k} \\
& \ddots & \\
\star & & \Lambda_{66 j k}
\end{array}\right]<0 ; j=1, \ldots, N, k=1, \ldots, N, j \neq k
$$

com

$$
\begin{aligned}
& \Lambda_{11 j k}=2\left(F_{1 j}+F_{1 j}^{\prime}\right)+F_{1 k}+F_{1 k}^{\prime} \\
& \Lambda_{12 j k}=-F_{1 j} A_{j}-F_{1 j} A_{k}-F_{1 k} A_{j}-\left(F_{1 j}+N_{1 j}\right) A_{\tau j}-\left(F_{1 j}+N_{1 j}\right) A_{\tau k}-\left(F_{1 k}+N_{1 k}\right) A_{\tau j} \\
& -G_{1 j} A_{\tau j} A_{k}-G_{1 j} A_{\tau k} A_{j}-G_{1 k} A_{\tau j} A_{j}-2 H_{1 j}-H_{1 k}+2 F_{2 j}+F_{2 k}^{\prime}+2 P_{j}+P_{k} \\
& \Lambda_{13 j k}=2\left(G_{1 j}+F_{3 j}^{\prime}\right)+G_{1 k}+F_{3 k}^{\prime} ; \Lambda_{14 j k}=2\left(H_{1 j}+F_{4 j}^{\prime}\right)+H_{1 k}+F_{4 k}^{\prime} \\
& \Lambda_{15 j k}=2\left(-\bar{\tau} F_{1 j}-\bar{\tau} N_{1 j}+F_{5 j}^{\prime}\right)-\bar{\tau} F_{1 k}-\bar{\tau} N_{1 k}+F_{5 k}^{\prime} \\
& \Lambda_{16 j k}=N_{1 j} A_{\tau j}+N_{1 j} A_{\tau k}+N_{1 k} A_{\tau j}-G_{1 j} A_{\tau j} A_{\tau k}-G_{1 j} A_{\tau k} A_{\tau j}-G_{1 k} A_{\tau j} A_{\tau j}+2 F_{6 j}^{\prime}+F_{6 k}^{\prime} \\
& \Lambda_{22 j k}=-F_{2 j} A_{j}-F_{2 j} A_{k}-F_{2 k} A_{j}-A_{j}^{\prime} F_{2 j}^{\prime}-A_{j}^{\prime} F_{2 k}^{\prime}-A_{k}^{\prime} F_{2 j}^{\prime}-\left(F_{2 j}+N_{2 j}\right) A_{\tau j}-\left(F_{2 j}+N_{2 j}\right) A_{\tau k} \\
& -\left(F_{2 k}+N_{2 k}\right) A_{\tau j}-A_{\tau j}^{\prime}\left(F_{2 j}^{\prime}+N_{2 j}^{\prime}\right)-A_{\tau j}^{\prime}\left(F_{2 k}^{\prime}+N_{2 k}^{\prime}\right)-A_{\tau k}^{\prime}\left(F_{2 j}^{\prime}+N_{2 j}^{\prime}\right) \\
& -G_{2 j} A_{\tau j} A_{k}-G_{2 j} A_{\tau k} A_{j}-G_{2 k} A_{\tau k} A_{j}-A_{j}^{\prime} A_{\tau j}^{\prime} G_{2 k}^{\prime}-A_{j}^{\prime} A_{\tau k}^{\prime} G_{2 j}^{\prime}-A_{k}^{\prime} A_{\tau j}^{\prime} G_{2 j}^{\prime}-2 H_{3 j}-H_{3 k}-2 H_{3 j}^{\prime}-H_{3 k}^{\prime} \\
& \Lambda_{23 j k}=2 G_{2 j}+G_{2 k}-A_{j}^{\prime} F_{3 j}^{\prime}-A_{j}^{\prime} F_{3 k}^{\prime}-A_{k}^{\prime} F_{3 j}^{\prime}-A_{\tau j}^{\prime}\left(N_{3 j}^{\prime}+F_{3 j}^{\prime}\right)-A_{\tau j}^{\prime}\left(N_{3 k}^{\prime}+F_{3 k}^{\prime}\right)-A_{\tau k}^{\prime}\left(N_{3 j}^{\prime}+F_{3 j}^{\prime}\right) \\
& -A_{j}^{\prime} A_{\tau j}^{\prime} G_{3 k}^{\prime}-A_{j}^{\prime} A_{\tau k}^{\prime} G_{3 j}^{\prime}-A_{k}^{\prime} A_{\tau j}^{\prime} G_{3 j}^{\prime}-2 H_{3 j}^{\prime}-H_{3 k}^{\prime} \\
& \Lambda_{24 j k}=2 H_{3 j}+H_{3 k}-A_{j}^{\prime} F_{4 j}^{\prime}-A_{j}^{\prime} F_{4 k}^{\prime}-A_{k}^{\prime} F_{4 j}^{\prime}-A_{\tau j}^{\prime}\left(N_{4 j}^{\prime}+G_{4 j}^{\prime}\right)-A_{\tau j}^{\prime}\left(N_{4 k}^{\prime}+G_{4 k}^{\prime}\right)-A_{\tau k}^{\prime}\left(N_{4 j}^{\prime}+G_{4 j}^{\prime}\right) \\
& -A_{j}^{\prime} A_{\tau j}^{\prime} G_{4 k}^{\prime}-A_{j}^{\prime} A_{\tau k}^{\prime} G_{4 j}^{\prime}-A_{k}^{\prime} A_{\tau j}^{\prime} G_{4 j}^{\prime}-2 H_{4 j}^{\prime}-H_{4 k}^{\prime} \\
& \Lambda_{25 j k}=-2 \bar{\tau} F_{2 j}-\bar{\tau} F_{2 k}-2 \bar{\tau} N_{2 j}-\bar{\tau} N_{2 k}-A_{j}^{\prime} F_{5 j}^{\prime}-A_{j}^{\prime} F_{5 k}^{\prime}-A_{k}^{\prime} F_{5 j}^{\prime}-A_{\tau j}^{\prime}\left(N_{5 j}^{\prime}+F_{5 j}^{\prime}\right)-A_{\tau j}^{\prime}\left(N_{5 k}^{\prime}+F_{5 k}^{\prime}\right) \\
& -A_{\tau k}^{\prime}\left(N_{5 j}^{\prime}+F_{5 j}^{\prime}\right)-A_{j}^{\prime} A_{\tau j}^{\prime} G_{5 k}^{\prime}-A_{j}^{\prime} A_{\tau k}^{\prime} G_{5 j}^{\prime}-A_{k}^{\prime} A_{\tau j}^{\prime} G_{5 j}^{\prime}-2 H_{5 j}^{\prime}-H_{5 j}^{\prime} \\
& \Lambda_{26 j k}=-N_{2 j} A_{\tau j}-N_{2 j} A_{\tau k}-N_{2 k} A_{\tau j}-G_{2 j} A_{\tau j} A_{\tau k}-G_{2 j} A_{\tau k} A_{\tau j}-G_{2 k} A_{\tau j} A_{\tau j}-A_{j}^{\prime} F_{6 j}^{\prime}-A_{j}^{\prime} F_{6 k}^{\prime}-A_{k}^{\prime} F_{6 j}^{\prime} \\
& -A_{\tau j}^{\prime}\left(N_{6 j}^{\prime}+F_{6 j}^{\prime}\right)-A_{\tau j}^{\prime}\left(N_{6 k}^{\prime}+F_{6 k}^{\prime}\right)-A_{\tau k}^{\prime}\left(N_{6 j}^{\prime}+F_{6 j}^{\prime}\right)-A_{j}^{\prime} A_{\tau j}^{\prime} G_{6 k}^{\prime}-A_{j}^{\prime} A_{\tau k}^{\prime} G_{6 j}^{\prime}-A_{k}^{\prime} A_{\tau j}^{\prime} G_{6 j}^{\prime}-2 H_{6 j}^{\prime}-H_{6 j}^{\prime}
\end{aligned}
$$

$\Lambda_{33 j k}=2 G_{3 j}+G_{3 k}+2 G_{3 j}^{\prime}+G_{3 k}^{\prime}+2 V_{j}+V_{k} ; \Lambda_{34 j k}=2 H_{3 j}+H_{3 k}+2 G_{4 j}^{\prime}+G_{4 k}^{\prime} ; \Lambda_{35 j k}=-2 \bar{\tau} F_{3 j}-\bar{\tau} F_{3 k}-2 \bar{\tau} N_{3 j}-\bar{\tau} N_{3 k}+2 G_{5 j}^{\prime}+G_{5 k}^{\prime}$

$\Lambda_{36 j k}=N_{3 j} A_{\tau j}+N_{3 j} A_{\tau k}+N_{3 k} A_{\tau j}-G_{3 j} A_{\tau j} A_{\tau k}-G_{3 j} A_{\tau k} A_{\tau j}-G_{3 k} A_{\tau j} A_{\tau j}+2 G_{6 j}^{\prime}+G_{6 k}^{\prime}$

$\Lambda_{44 j k}=2 H_{4 j}+H_{4 k}+2 H_{4 j}^{\prime}+H_{4 k}^{\prime}+2 U_{j}+U_{k} ; \Lambda_{45 j}=-2 \bar{\tau} F_{4 j}-\bar{\tau} F_{4 k}-2 \bar{\tau} N_{4 j}-\bar{\tau} N_{4 k}+2 H_{5 j}^{\prime}+H_{5 k}^{\prime}$

$\Lambda_{46 j k}=N_{3 j} A_{\tau j}+N_{3 j} A_{\tau k}+N_{3 k} A_{\tau j}-G_{3 j} A_{\tau j} A_{\tau k}-G_{3 j} A_{\tau k} A_{\tau j}-G_{3 k} A_{\tau j} A_{\tau j}+2 G_{6 j}^{\prime}+G_{6 k}^{\prime}$

$\Lambda_{55 j}=-2 \bar{\tau} F_{5 j}-\bar{\tau} F_{5 k}-2 \bar{\tau} N_{5 j}-\bar{\tau} N_{5 k}-2 \bar{\tau} F_{5 j}^{\prime}-\bar{\tau} F_{5 k}^{\prime}-2 \bar{\tau} N_{5 j}^{\prime}-\bar{\tau} N_{5 k}^{\prime}-2 V_{k}-V_{j}$

$\Lambda_{56 j}=N_{5 j} A_{\tau j}+N_{5 j} A_{\tau k}+N_{5 k} A_{\tau j}-G_{5 j} A_{\tau j} A_{\tau k}-G_{5 j} A_{\tau k} A_{\tau j}-G_{5 k} A_{\tau j} A_{\tau j}-2 \bar{\tau}\left(F_{6 j}^{\prime}+N_{6 j}^{\prime}\right)-\bar{\tau}\left(F_{6 k}^{\prime}+N_{6 k}^{\prime}\right)$

$\Lambda_{66 j}=-N_{6 j} A_{\tau j}-N_{6 j} A_{\tau k}-N_{6 k} A_{\tau j}-G_{6 j} A_{\tau j} A_{\tau k}-G_{6 j} A_{\tau k} A_{\tau j}-G_{6 k} A_{\tau j} A_{\tau j}+A_{\tau j}^{\prime} N_{6 j}^{\prime}+A_{\tau j}^{\prime} N_{6 k}^{\prime}+A_{\tau k}^{\prime} N_{6 j}^{\prime}$

$$
-A_{\tau k}^{\prime} A_{\tau j}^{\prime} G_{6 j}^{\prime}-A_{\tau j}^{\prime} A_{\tau k}^{\prime} G_{6 j}^{\prime}-A_{\tau k}^{\prime} A_{\tau j}^{\prime} G_{6 j}^{\prime}-2 U_{j}-U_{k}
$$

Tabela 1: Valores de $K$ (variáveis escalares) e $L$ (número de linhas de LMIs) para os lemas 3 a 11.

\begin{tabular}{c|c|c} 
Lema & $K$ & $L$ \\
\hline 3 & $n(n+1) / 2+8 n^{2}$ & $6 n$ \\
4 & $(3 n(n+1) / 2)+18 n^{2}$ & $9 n$ \\
5 & $(3 n(n+1) / 2)+24 n^{2}$ & $9 n$ \\
6 & $N(n(n+1))$ & $3 n(N+N(N-1) / 2)+2 n N$ \\
7 & $N\left(n(n+1)+8 n^{2}\right)$ & $4 n(N+N(N-1) / 2)+2 n N$ \\
8 & $N(3 n(n+1) / 2)$ & $5 n(N+N(N-1) / 2)+3 n N$ \\
9 & $N\left(3 n(n+1) / 2+18 n^{2}\right)$ & $6 n(N+N(N-1) / 2)+3 n N$ \\
10 & $N\left(3 n(n+1) / 2+n^{2}\right)$ & $5 n\left(N+N(N-1)+\left(N^{3}-3 N^{2}+2 N\right) / 6\right)+3 n N$ \\
11 & $N\left(3 n(n+1) / 2+24 n^{2}\right)$ & $6 n\left(N+N(N-1)+\left(N^{3}-3 N^{2}+2 N\right) / 6\right)+3 n N$
\end{tabular}


com

$$
\left[\begin{array}{ccc}
\Lambda_{11 j k \ell} & \cdots & \Lambda_{16 j k \ell} \\
& \ddots & \\
\star & & \Lambda_{66 j k \ell}
\end{array}\right]<0 ; j=1, \ldots, N-2, k=j+1, \ldots, N-1, \ell=k+1, \ldots, N
$$

$$
\begin{aligned}
& \Lambda_{11 j k \ell}=2\left(F_{1 j}+F_{1 k}+F_{1 \ell}\right)+2\left(F_{1 j}^{\prime}+F_{1 k}^{\prime}+F_{1 \ell}^{\prime}\right) ; \\
& \Lambda_{12 j k \ell}=-F_{1 j} A_{k}-F_{1 j} A_{\ell}-F_{1 k} A_{j}-F_{1 k} A_{\ell}-F_{1 \ell} A_{j}-F_{1 \ell} A_{k}-\left(F_{1 j}+N_{1 j}\right) A_{\tau k}-\left(F_{1 j}+N_{1 j}\right) A_{\tau \ell}-\left(F_{1 k}+N_{1 k}\right) A_{\tau j} \\
& -\left(F_{1 k}+N_{1 k}\right) A_{\tau \ell}-\left(F_{1 \ell}+N_{1 \ell}\right) A_{\tau j}-\left(F_{1 \ell}+N_{1 \ell}\right) A_{\tau k}-G_{1 j} A_{\tau k} A_{\ell}-G_{1 j} A_{\tau \ell} A_{k}-G_{1 k} A_{\tau j} A_{\ell}-G_{1 k} A_{\tau \ell} A_{j}-G_{1 \ell} A_{\tau j} A_{k}-G_{1 \ell} A_{\tau k} A_{j} \\
& -2 H_{1 j}-2 H_{1 k}-2 H_{1 \ell}+2 F_{2 j}^{\prime}+2 F_{2 j}^{\prime}+2 F_{2 \ell}^{\prime}+2 P_{j}+2 P_{k}+2 P_{\ell} \\
& \Lambda_{13 j k \ell}=2 G_{1 j}+2 G_{1 k}+2 G_{1 \ell}+2 F_{3 j}^{\prime}+2 F_{3 k}^{\prime}+2 F_{3 \ell}^{\prime} ; \Lambda_{14 j k \ell}=2 H_{1 j}+2 F_{4 j}^{\prime}+2 H_{1 k}+2 F_{4 k}^{\prime}+2 H_{1 \ell}+2 F_{4 \ell}^{\prime} \\
& \Lambda_{15 j k \ell}=-2 \bar{\tau} F_{1 j}-2 \bar{\tau} N_{1 j}+2 F_{5 j}^{\prime}-2 \bar{\tau} F_{1 k}-2 \bar{\tau} N_{1 k}+2 F_{5 k}^{\prime}-2 \bar{\tau} F_{1 \ell}-2 \bar{\tau} N_{1 \ell}+2 F_{5 \ell}^{\prime} \\
& \Lambda_{16 j k \ell}=N_{1 j} A_{\tau k}+N_{1 j} A_{\tau \ell}+N_{1 k} A_{\tau j}+N_{1 k} A_{\tau \ell}+N_{1 \ell} A_{\tau j}+N_{1 \ell} A_{\tau k} \\
& -G_{1 j} A_{\tau k} A_{\tau \ell}-G_{1 j} A_{\tau \ell} A_{\tau k}-G_{1 k} A_{\tau j} A_{\tau \ell}-G_{1 k} A_{\tau \ell} A_{\tau j}-G_{1 \ell} A_{\tau j} A_{\tau k}-G_{1 \ell} A_{\tau k} A_{\tau j}+2 F_{6 j}^{\prime}+2 F_{6 k}^{\prime}+2 F_{6 \ell}^{\prime} \\
& \Lambda_{22 j k \ell}=-F_{2 j} A_{k}-F_{2 j} A_{\ell}-F_{2 k} A_{j}-F_{2 k} A_{\ell}-F_{2 \ell} A_{j}-F_{2 \ell} A_{k}-A_{j}^{\prime} F_{2 k}^{\prime}-A_{j}^{\prime} F_{2 \ell}^{\prime}-A_{k}^{\prime} F_{2 j}^{\prime}-A_{k}^{\prime} F_{2 \ell}^{\prime}-A_{\ell}^{\prime} F_{2 j}^{\prime}-A_{\ell}^{\prime} F_{2 k}^{\prime} \\
& -\left(F_{2 j}+N_{2 j}\right) A_{\tau k}-\left(F_{2 k}+N_{2 k}\right) A_{\tau j}-\left(F_{2 j}+N_{2 j}\right) A_{\tau \ell}-\left(F_{2 \ell}+N_{2 \ell}\right) A_{\tau j}-\left(F_{2 k}+N_{2 k}\right) A_{\tau \ell}-\left(F_{2 \ell}+N_{2 \ell}\right) A_{\tau k}-A_{\tau j}^{\prime}\left(F_{2 k}^{\prime}+N_{2 k}^{\prime}\right) \\
& -A_{\tau j}^{\prime}\left(F_{2 \ell}^{\prime}+N_{2 \ell}^{\prime}\right)-A_{\tau k}^{\prime}\left(F_{2 j}^{\prime}+N_{2 j}^{\prime}\right)-A_{\tau j}^{\prime}\left(F_{2 \ell}^{\prime}+N_{2 \ell}^{\prime}\right)-A_{\tau k}^{\prime}\left(F_{2 \ell}^{\prime}+N_{2 \ell}^{\prime}\right)-A_{\tau \ell}^{\prime}\left(F_{2 k}^{\prime}+N_{2 k}^{\prime}\right)-G_{2 j} A_{\tau k} A_{\ell}-G_{2 j} A_{\tau \ell} A_{k}-G_{2 k} A_{\tau j} A_{\ell} \\
& -G_{2 k} A_{\tau \ell} A_{j}-G_{2 \ell} A_{\tau j} A_{k}-G_{2 \ell} A_{\tau j} A_{k}-A_{j}^{\prime} A_{\tau k}^{\prime} G_{2 \ell}^{\prime}-A_{j}^{\prime} A_{\tau \ell}^{\prime} G_{2 k}^{\prime}-A_{k}^{\prime} A_{\tau j}^{\prime} G_{2 \ell}^{\prime}-A_{k}^{\prime} A_{\tau \ell}^{\prime} G_{2 j}^{\prime}-A_{\ell}^{\prime} A_{\tau j}^{\prime} G_{2 k}^{\prime}-A_{\ell}^{\prime} A_{\tau k}^{\prime} G_{2 j}^{\prime} \\
& -2 H_{3 j}-2 H_{3 k}-2 H_{3 \ell}-2 H_{3 j}^{\prime}-2 H_{3 k}^{\prime}-2 H_{3 \ell}^{\prime} \\
& \Lambda_{23 j k \ell}=2 G_{2 j}+2 G_{2 k}+2 G_{2 \ell}-A_{j}^{\prime} F_{3 k}^{\prime}-A_{k}^{\prime} F_{3 j}^{\prime}-A_{j}^{\prime} F_{3 \ell}^{\prime}-A_{\ell}^{\prime} F_{3 j}^{\prime}-A_{k}^{\prime} F_{3 \ell}^{\prime}-A_{\ell}^{\prime} F_{3 k}^{\prime}-A_{\tau j}^{\prime}\left(N_{3 j}^{\prime}+F_{3 j}^{\prime}\right)-A_{\tau j}^{\prime}\left(N_{3 k}^{\prime}+F_{3 k}^{\prime}\right) \\
& -A_{\tau k}^{\prime}\left(N_{3 j}^{\prime}+F_{3 j}^{\prime}\right)-A_{\tau j}^{\prime}\left(N_{3 \ell}^{\prime}+F_{3 \ell}^{\prime}\right)-A_{\tau \ell}^{\prime}\left(N_{3 j}^{\prime}+F_{3 j}^{\prime}\right)-A_{\tau k}^{\prime}\left(N_{3 \ell}^{\prime}+F_{3 \ell}^{\prime}\right)-A_{\tau \ell}^{\prime}\left(N_{3 k}^{\prime}+F_{3 k}^{\prime}\right)-A_{j}^{\prime} A_{\tau k}^{\prime} G_{3 \ell}^{\prime} \\
& -A_{j}^{\prime} A_{\tau \ell}^{\prime} G_{3 k}^{\prime}-A_{k}^{\prime} A_{\tau j}^{\prime} G_{3 \ell}^{\prime}-A_{k}^{\prime} A_{\tau \ell}^{\prime} G_{3 j}^{\prime}-A_{\ell}^{\prime} A_{\tau j}^{\prime} G_{3 k}^{\prime}-A_{\ell}^{\prime} A_{\tau k}^{\prime} G_{3 j}^{\prime}-2 H_{3 j}^{\prime}-2 H_{3 k}^{\prime}-2 H_{3 \ell}^{\prime} \\
& \Lambda_{24 j k \ell}=H_{3 j}-A_{j}^{\prime} F_{4 j}^{\prime}-A_{\tau j}^{\prime}\left(N_{4 j}^{\prime}+G_{4 j}^{\prime}\right)-A_{j}^{\prime} A_{\tau j}^{\prime} G_{4 j}^{\prime}-H_{4 j}^{\prime}+H_{3 k}-A_{k}^{\prime} F_{4 k}^{\prime}-A_{\tau k}^{\prime}\left(N_{4 k}^{\prime}+G_{4 k}^{\prime}\right)-A_{k}^{\prime} A_{\tau k}^{\prime} G_{4 k}^{\prime}-H_{4 k}^{\prime} \\
& +H_{3 \ell}-A_{\ell}^{\prime} F_{4 \ell}^{\prime}-A_{\tau \ell}^{\prime}\left(N_{4 \ell}^{\prime}+G_{4 \ell}^{\prime}\right)-A_{\ell}^{\prime} A_{\tau \ell}^{\prime} G_{4 \ell}^{\prime}-H_{4 \ell}^{\prime} \\
& \Lambda_{25 j k \ell}=-2 \bar{\tau} F_{2 j}-2 \bar{\tau} F_{2 k}-2 \bar{\tau} F_{2 \ell}-2 \bar{\tau} N_{2 j}-2 \bar{\tau} N_{2 k}-2 \bar{\tau} N_{2 \ell}-A_{j}^{\prime} F_{5 k}^{\prime}-A_{k}^{\prime} F_{5 j}^{\prime}-A_{j}^{\prime} F_{5 \ell}^{\prime}-A_{\ell}^{\prime} F_{5 j}^{\prime}-A_{k}^{\prime} F_{5 \ell}^{\prime}-A_{\ell}^{\prime} F_{5 k}^{\prime} \\
& -A_{\tau j}^{\prime}\left(N_{5 k}^{\prime}+F_{5 k}^{\prime}\right)-A_{\tau k}^{\prime}\left(N_{5 j}^{\prime}+F_{5 j}^{\prime}\right)-A_{\tau j}^{\prime}\left(N_{5 \ell}^{\prime}+F_{5 \ell}^{\prime}\right)-A_{\tau \ell}^{\prime}\left(N_{5 j}^{\prime}+F_{5 j}^{\prime}\right)-A_{\tau k}^{\prime}\left(N_{5 \ell}^{\prime}+F_{5 \ell}^{\prime}\right)-A_{\tau \ell}^{\prime}\left(N_{5 k}^{\prime}+F_{5 k j}^{\prime}\right)-A_{j}^{\prime} A_{\tau k}^{\prime} G_{5 \ell}^{\prime} \\
& -A_{j}^{\prime} A_{\tau \ell}^{\prime} G_{5 k}^{\prime}-A_{k}^{\prime} A_{\tau j}^{\prime} G_{5 \ell}^{\prime}-A_{k}^{\prime} A_{\tau \ell}^{\prime} G_{5 j}^{\prime}-A_{\ell}^{\prime} A_{\tau j}^{\prime} G_{5 k}^{\prime}-A_{\ell}^{\prime} A_{\tau k}^{\prime} G_{5 j}^{\prime}-2 H_{5 j}^{\prime}-2 H_{5 k}^{\prime}-2 H_{5 \ell}^{\prime} \\
& \Lambda_{26 j k \ell}=-N_{2 j} A_{\tau k}-N_{2 k} A_{\tau j}-N_{2 j} A_{\tau \ell}-N_{2 \ell} A_{\tau j}-N_{2 k} A_{\tau \ell}-N_{2 \ell} A_{\tau k}-G_{2 j} A_{\tau k} A_{\tau \ell}-G_{2 j} A_{\tau \ell} A_{\tau k}-G_{2 k} A_{\tau j} A_{\tau \ell}-G_{2 k} A_{\tau \ell} A_{\tau j} \\
& -G_{2 \ell} A_{\tau j} A_{\tau k}-G_{2 \ell} A_{\tau k} A_{\tau j}-A_{j}^{\prime} F_{6 k}^{\prime}-A_{k}^{\prime} F_{6 j}^{\prime}-A_{j}^{\prime} F_{6 \ell}^{\prime}-A_{\ell}^{\prime} F_{6 j}^{\prime}-A_{k}^{\prime} F_{6 \ell}^{\prime}-A_{\ell}^{\prime} F_{6 k}^{\prime}-A_{\tau j}^{\prime}\left(N_{6 k}^{\prime}+F_{6 k}^{\prime}\right)-A_{\tau k}^{\prime}\left(N_{6 j}^{\prime}+F_{6 j}^{\prime}\right) \\
& -A_{\tau j}^{\prime}\left(N_{6 \ell}^{\prime}+F_{6 \ell}^{\prime}\right)-A_{\tau \ell}^{\prime}\left(N_{6 j}^{\prime}+F_{6 j}^{\prime}\right)-A_{\tau k}^{\prime}\left(N_{6 \ell}^{\prime}+F_{6 \ell}^{\prime}\right)-A_{\tau \ell}^{\prime}\left(N_{6 k}^{\prime}+F_{6 k}^{\prime}\right)-A_{j}^{\prime} A_{\tau k}^{\prime} G_{6 \ell}^{\prime}-A_{j}^{\prime} A_{\tau \ell}^{\prime} G_{6 k}^{\prime}-A_{k}^{\prime} A_{\tau j}^{\prime} G_{6 \ell}^{\prime}-A_{k}^{\prime} A_{\tau \ell}^{\prime} G_{6 j}^{\prime} \\
& -A_{\ell}^{\prime} A_{\tau j}^{\prime} G_{6 k}^{\prime}-A_{\ell}^{\prime} A_{\tau k}^{\prime} G_{6 j}^{\prime}-2 H_{6 j}^{\prime}-2 H_{6 k}^{\prime}-2 H_{6 \ell}^{\prime} \\
& -G_{3 k} A_{\tau \ell} A_{\tau j}-G_{3 \ell} A_{\tau j} A_{\tau k}-G_{3 \ell} A_{\tau k} A_{\tau j}+2 G_{6 j}^{\prime}+2 G_{6 k}^{\prime}+2 G_{6 \ell}^{\prime} \\
& \Lambda_{44 j k \ell}=2 H_{4 j}+2 H_{4 k}+2 H_{4 \ell}+2 H_{4 j}^{\prime}+2 H_{4 k}^{\prime}+2 H_{4 \ell}^{\prime}+2 U_{j}+2 U_{k}+2 U_{\ell} \\
& \Lambda_{45 j k \ell}=-2 \bar{\tau} F_{4 j}-2 \bar{\tau} F_{4 k}-2 \bar{\tau} F_{4 \ell}-2 \bar{\tau} N_{4 j}-2 \bar{\tau} N_{4 k}-2 \bar{\tau} N_{4 \ell}+2 H_{5 j}^{\prime}+2 H_{5 k}^{\prime}+2 H_{5 \ell}^{\prime} \\
& \Lambda_{46 j k \ell}=N_{4 j} A_{\tau k}+N_{4 k} A_{\tau j}+N_{4 j} A_{\tau \ell}+N_{4 \ell} A_{\tau j}+N_{4 k} A_{\tau \ell}+N_{4 \ell} A_{\tau k}-G_{4 j} A_{\tau k} A_{\tau \ell}-G_{4 j} A_{\tau \ell} A_{\tau k} \\
& -G_{4 k} A_{\tau j} A_{\tau \ell}-G_{4 k} A_{\tau \ell} A_{\tau j}-G_{4 \ell} A_{\tau j} A_{\tau k}-G_{4 \ell} A_{\tau k} A_{\tau j}+2 H_{6 j}^{\prime}+2 H_{6 k}^{\prime}+2 H_{6 \ell}^{\prime}
\end{aligned}
$$




$$
\begin{aligned}
& \left(A, A_{\tau}\right)_{1}=\left(\left[\begin{array}{ccc}
-30.761 & -308.96 & -449.269 \\
1 & 1.749 & 0 \\
0 & 1 & 0.749
\end{array}\right]\right. \text {, } \\
& \left.\left[\begin{array}{ccc}
0 & 10 & 20 \\
0 & 0 & 0 \\
0 & 0 & 3.749
\end{array}\right]\right) \\
& \begin{aligned}
\left(A, A_{\tau}\right)_{2}=\left(\left[\begin{array}{ccc}
-41.259 & -585.225 & -2347.695 \\
1 & 1.749 & 0 \\
0 & 1 & 0.749
\end{array}\right]\right. \\
\\
{\left.\left[\begin{array}{ccc}
0 & 10 & 130 \\
0 & 0 & 0 \\
0 & 0 & 3.749
\end{array}\right]\right) }
\end{aligned}
\end{aligned}
$$

Novamente, a condição com matrizes extras do Lema 7 identificou o sistema incerto como estável independentemente da magnitude do atraso, enquanto que as LMIs da condição sem variáveis extras são infactíveis para este sistema.

\section{CONCLUSÕES}

Foram apresentadas extensões de condições LMI do teorema do ganho pequeno escalonado para o caso de sistemas lineares contínuos com incerteza politópica. Para sistemas precisamente conhecidos (sem incerteza), as LMIs do teorema do ganho pequeno escalonado foram associadas na literatura a condições para a análise de estabilidade de sistemas com atraso, dependentes e independentes do atraso, obtidas por meio de funcionais de Lyapunov-Krasovskii. Neste trabalho, mostrou-se que condições equivalentes às condições do teorema do ganho pequeno escalonado podem ser expressas em um espaço paramétrico aumentado, com variáveis extras (multiplicadores) obtidas pela utilização do Lema de Finsler.

A partir de propriedades que exploram a positividade dos parâmetros incertos, foram obtidas condições suficientes que asseguram as condições do teorema do ganho pequeno escalonado para sistemas lineares com incertezas politópicas. As condições suficientes obtidas no espaço paramétrico aumentado são menos conservadoras que as condições suficientes no espaço original, pois as matrizes extras proporcionam graus de liberdade que melhoram as avaliações obtidas na análise de sistemas incertos. Assim como no caso precisamente conhecido, por meio da escolha particular das matrizes envolvidas nas LMIs das condições suficientes obtêm-se condições para a estabilidade robusta, dependente e independente do atraso, de sistemas lineares incertos com atrasos, tanto no espaço paramétrico original quanto no aumentado. Por meio de exemplos, foi mostrado que as avaliações com as LMIs com matrizes extras fornecem melhores resultados que as condições suficientes no espaço paramétrico original. No contexto de análise robusta de sistemas com atrasos, as condições de estabilidade robusta garantem a existência de funcionais com matrizes dependentes de parâmetros, proporcionando resultados menos conservadores do que os obtidos com matrizes constantes.

No espaço aumentado de parâmetros, ocorre a separação das matrizes que descrevem a dinâmica do sistema das matrizes que compõem os funcionais usados na avaliação da estabilidade robusta. Uma possível extensão deste trabalho seria a síntese de controladores robustos para sistemas com atrasos baseados nas variáveis matriciais introduzidas pelo Lema de Finsler, mantendo os funcionais dependentes de parâmetros, ou mesmo a síntese de controladores dependentes de parâmetros.

\section{AGRADECIMENTOS}

Este trabalho contou com o apoio das agências CAPES, CNPq, FAPEMIG (TEC 840/05) e FAPESP. Os autores agradecem os comentários e as sugestões dos revisores anônimos.

\section{REFERÊNCIAS}

Boyd, S., El Ghaoui, L., Feron, E. e Balakrishnan, V. (1994). Linear Matrix Inequalities in System and Control Theory, SIAM Studies in Applied Mathematics, Philadelphia, PA.

de Oliveira, M. C., Bernussou, J. e Geromel, J. C. (1999). A new discrete-time robust stability condition, Systems \& Control Letters 37(4): 261-265.

de Oliveira, M. C. e Skelton, R. E. (2001). Stability tests for constrained linear systems, in S. O. Reza Moheimani (ed.), Perspectives in Robust Control, Vol. 268 of Lecture Notes in Control and Information Science, Springer-Verlag, New York, pp. 241-257.

Gahinet, P., Nemirovskii, A., Laub, A. J. e Chilali, M. (1995). LMI Control Toolbox User's Guide, The Math Works Inc., Natick, MA.

Gu, K., Kharitonov, V. L. e Chen, J. (2003). Stability of Time-delay Systems, Control Engineering, Birkhäuser, Boston, MA.

Huang, Y. e Zhou, K. (1999). Robust control of uncertain time delay systems, Proceedings of the 38th IEEE Conference on Decision and Control, pp. 1130-1135. 
Leite, V. J. S. e Peres, P. L. D. (2003). An improved LMI condition for robust $\mathscr{D}$-stability of uncertain polytopic systems, IEEE Transactions on Automatic Control 48(3): 500-504.

Li, X. e de Souza, C. E. (1995). LMI approach to delaydependent robust stability and stabilization of uncertain linear delay systems, Proceedings of the 34th IEEE Conference on Decision and Control, pp. 3614-3619.

Li, X. e de Souza, C. E. (1996). Robust stabilization and $\mathscr{H}_{\infty}$ of uncertain linear time-delay systems, Proceedings of the 13th IFAC World Congress, Vol. H, San Francisco, CA, pp. 113-118.

Mahmoud, M. S. (2000). Robust Control and Filtering for Time-Delay Systems, Control Engineering Series, Marcel Dekker, Inc., New York.

Niculescu, S.-I. (2001). On delay-dependent stability under model transformations of some neutral linear systems, International Journal of Control 74(6): 609-617.

Niculescu, S.-I. e Chen, J. (1999). Frequency sweeping tests for asymptotic stability: a model transformation for multiple delays, Proceedings of the 38th IEEE Conference on Decision and Control, pp. 4678-4683.

Niculescu, S.-I., Neto, A. T., Dion, J.-M. e Dugard, L. (1995). Delay-dependent stability of linear systems with delayed state: An LMI approach, Proceedings of the 34th IEEE Conference on Decision and Control, pp. 1495-1497.

Park, P. (1999). A delay-dependent stability criterion for systems with uncertain time-invariant delays, IEEE Transactions on Automatic Control 44(3): 876-487.

Peaucelle, D., Arzelier, D., Bachelier, O. e Bernussou, J. (2000). A new robust $\mathscr{D}$-stability condition for real convex polytopic uncertainty, Systems \& Control Letters 40(1): 21-30.

Peres, P. L. D., Tarbouriech, S., Garcia, G. e Leite, V. J. S. (2003). Robust stability of time-delay continuous-time systems in polytopic domains, Proceedings of the 2003 European Control Conference, Cambridge, UK. in CDrom.

Ramos, D. C. W. e Peres, P. L. D. (2001). A less conservative LMI condition for the robust stability of discretetime uncertain systems, Systems \& Control Letters 43(5): 371-378.

Ramos, D. C. W. e Peres, P. L. D. (2002). An LMI condition for the robust stability of uncertain continuous-time linear systems, IEEE Transactions on Automatic Control 47(4): 675-678.
Richard, J.-P. (2003). Time-delay systems: an overview of some recent advances and open problems, Automatica 39(10): 1667-1694.

Sturm, J. F. (1999). Using SeDuMi 1.02, a MATLAB toolbox for optimization over symmetric cones, Optimization Methods and Software 11-12: 625-653. URL: http://sedumi.mcmaster.ca/.

Valmórbida, G. (2006). Estabilidade de sistemas com atraso: Análise de incertezas e de saturação empregando desigualdades matriciais lineares, Master's thesis, Universidade Estadual de Campinas, Campinas, SP, Brazil.

Verriest, E. I., Fan, M. K. H. e Kullstam, J. (1993). Frequency domain robust stability criteria for linear delay systems, Proceedings of the 32nd IEEE Conference on Decision and Control, pp. 3473-3478.

Zhang, J., Knospe, C. e Tsiotras, P. (2001). Stability of time-delay systems: equivalence between Lyapunov and scaled small-gain conditions, IEEE Transactions on Automatic Control 46(3): 482-486.

Zhang, X., Tsiotras, P. e Knospe, C. (2002). Stability analysis of LPV time-delayed systems, International Journal of Control 75(7): 538-558. 